\title{
R Codes and Appendix
}

\author{
Supporting Information \\ for the manuscript "Statistical determination of synergy based on Bliss definition of \\ drugs independence" \\ by Eugene Demidenko and Todd W. Miller
}

The aim of this supplement is to provide complete $\mathrm{R}$ codes to computations in the paper along with the required data. The public domain $R$ package is freely available at https://www. R-project.org. The following *.csv data files are required/provided: Daphnia.csv, ZR75.csv and MCF-7_April_2016.csv. These data files must be stored in the folders specified by each program at the beginning of the code (the folders may be changed).

\section{Treatment interactions with replicates}

The Excel file in the .csv format with surviving fraction (SF) data for three treatment groups A, B, and AB must have two columns: the first column is Treatment and the second SF. See file Daphnia.csv as an example and the screen snapshot below.

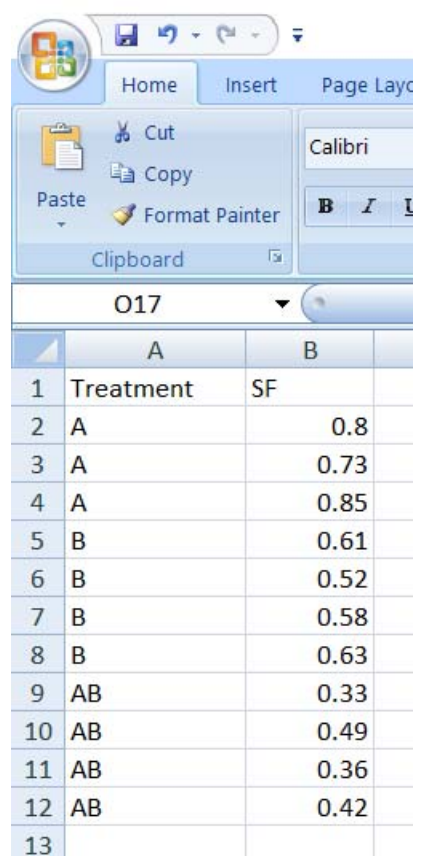

The $\mathrm{R}$ code for Daphnia example is listed below:

daphnia=function $(\mathrm{dr}=" \mathrm{c} ")$

\{

\#The folder with the data is : $\backslash \backslash$ Projects $\backslash \backslash$ ToddMiller $\backslash \backslash$ Synergy $\backslash \backslash$ Daphnia.csv da=read.csv (paste (dr, ": \\Projects $\backslash \backslash$ ToddMiller $\backslash \backslash$ Synergy $\backslash \backslash$ Daphnia.csv", sep=" ") )

\# names to be displayed

namgr=c $(" \mathrm{NiCl}=1.8 "$, "CuSO4=7", "NiCl=1.8 \& $\mathrm{CuSO} 4=7$ ", "Independence" $)$ 


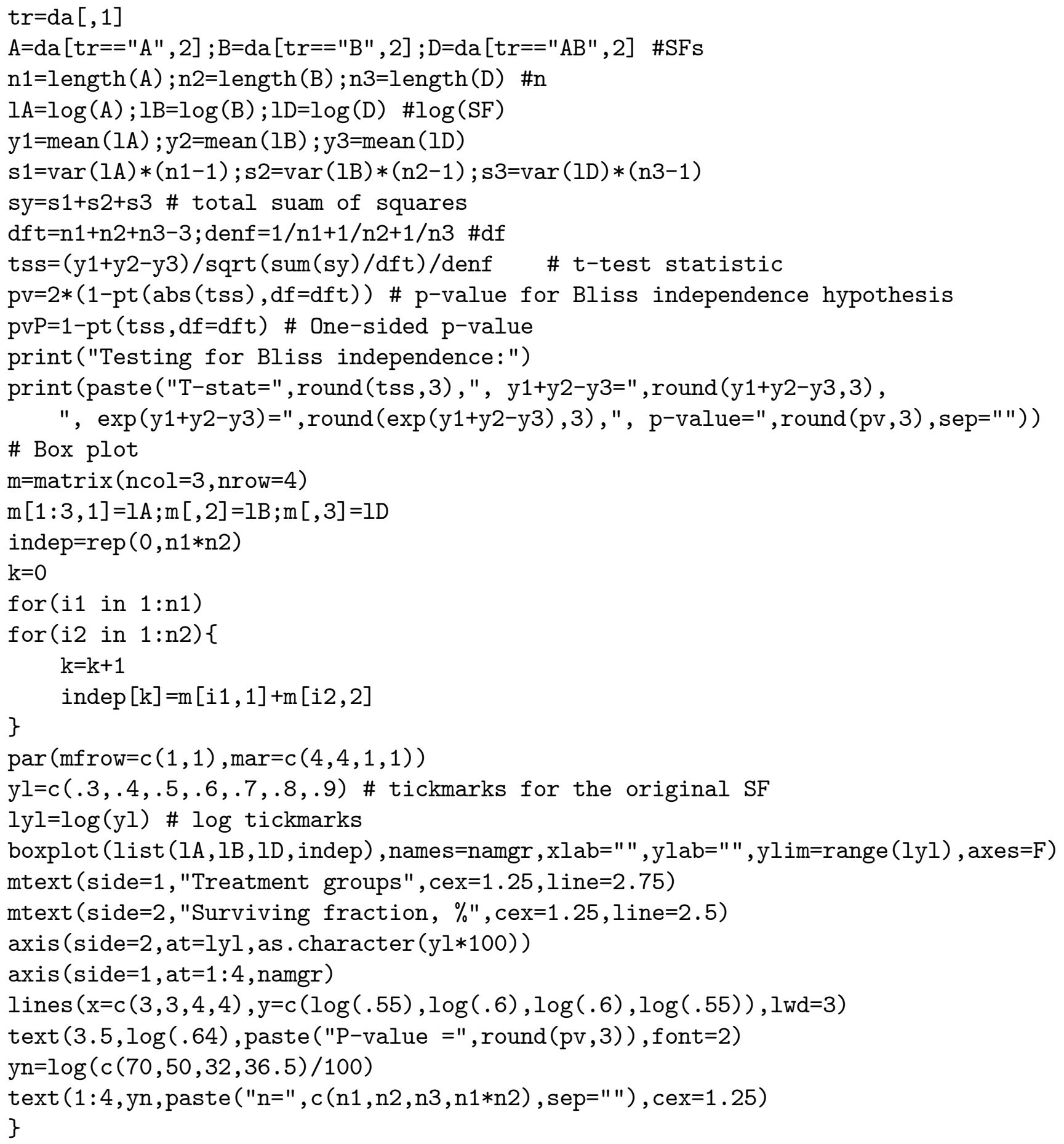

The call daphnia(dr="d") produces the folowing output (the data file Daphnia.csv must be saved in the folder specified by the read.csv command) and Figure 2:

$>$ daphnia $(\mathrm{dr}=" \mathrm{~d} ")$

[1] "Testing for Bliss independence:" 
[1] "T-stat=1.499, y1+y2-y3=0.156, $\exp (y 1+y 2-y 3)=1.168, p-v a l u e=0.172 "$

\section{Treatment interaction in the presence of control group}

The *.csv data file has the same format but contains information on the control group (C). The screen snapshot for the data from example on testing the synergy for cancer cells is shown below.

\begin{tabular}{|c|c|c|c|c|}
\hline (Es) & Home & Insert & \multicolumn{2}{|c|}{ Page I } \\
\hline \multirow{4}{*}{ Past } & \multirow{2}{*}{\multicolumn{2}{|c|}{$\begin{array}{l}\text { \& Cut } \\
\text { e Copy } \\
\text { Format Painter }\end{array}$}} & \multicolumn{2}{|c|}{ Calibri } \\
\hline & & & \multicolumn{2}{|c|}{ B $I$} \\
\hline & \multicolumn{2}{|l|}{ Clipboard } & & \\
\hline & \multicolumn{2}{|l|}{ K40 } & \multicolumn{2}{|c|}{ ( } \\
\hline 4 & A & \multicolumn{3}{|c|}{ B } \\
\hline 1 & Treatment & \multicolumn{3}{|l|}{ SF } \\
\hline 2 & C & \multicolumn{3}{|c|}{0.82927} \\
\hline 3 & C & \multicolumn{3}{|c|}{1.0662} \\
\hline 4 & C & \multicolumn{3}{|c|}{1.10105} \\
\hline 5 & A & \multicolumn{3}{|c|}{0.75958} \\
\hline 6 & A & \multicolumn{3}{|c|}{0.79443} \\
\hline 7 & A & \multicolumn{3}{|c|}{0.79791} \\
\hline 8 & B & \multicolumn{3}{|c|}{0.33798} \\
\hline 9 & B & \multicolumn{3}{|c|}{0.53659} \\
\hline 10 & B & \multicolumn{3}{|c|}{0.52613} \\
\hline 11 & $A B$ & \multicolumn{3}{|c|}{0.16028} \\
\hline 12 & $A B$ & \multicolumn{3}{|c|}{0.30662} \\
\hline 13 & $A B$ & \multicolumn{3}{|c|}{0.21254} \\
\hline 14 & & & & \\
\hline
\end{tabular}

The $\mathrm{R}$ code is similar to daphnia listed above.

bc. control=function ( $d r=" c ")$

\{

\#ZR75 cancer line cells

da $=$ read.csv (paste (dr, ": \\Projects $\backslash \backslash$ ToddMiller $\backslash \backslash$ Synergy $\backslash \backslash Z R 75 . c s v "$, sep=" "))

namgr=c ("BYL", "GSK", "BYL \& GSK", "Independence")

$\operatorname{tr}=\mathrm{da}[, 1]$

$\mathrm{C}=\mathrm{da}[\mathrm{tr}==" \mathrm{C} ", 2] ; \mathrm{A}=\mathrm{da}[\mathrm{tr}==" \mathrm{~A} ", 2] ; \mathrm{B}=\mathrm{da}[\mathrm{tr}==" \mathrm{~B} ", 2] ; \mathrm{D}=\mathrm{da}[\operatorname{tr}==" \mathrm{AB} ", 2]$ \#SFs

$\mathrm{n} 0=$ length $(\mathrm{C}) ; \mathrm{n} 1=$ length $(\mathrm{A}) ; \mathrm{n} 2=$ length $(\mathrm{B}) ; \mathrm{n} 3=$ length (D) \#n

$1 C=\log (C) ; 1 A=\log (A) ; 1 B=\log (B) ; 1 D=\log (D)$

$\mathrm{y} 0=$ mean $(1 C) ; \mathrm{y} 1=$ mean $(1 \mathrm{~A}) ; \mathrm{y} 2=$ mean $(1 \mathrm{~B}) ; \mathrm{y} 3=$ mean (ID)

$\mathrm{s} 0=\operatorname{var}(1 \mathrm{C}) *(\mathrm{n} 0-1) ; \mathrm{s} 1=\operatorname{var}(1 \mathrm{~A}) *(\mathrm{n} 1-1) ; \mathrm{s} 2=\operatorname{var}(1 \mathrm{~B}) *(\mathrm{n} 2-1) ; \mathrm{s} 3=\operatorname{var}(1 \mathrm{D}) *(\mathrm{n} 3-1)$

$\mathrm{sy}=\mathrm{s} 0+\mathrm{s} 1+\mathrm{s} 2+\mathrm{s} 3$

dft $=\mathrm{n} 0+\mathrm{n} 1+\mathrm{n} 2+\mathrm{n} 3-4 ; \operatorname{denf}=1 / \mathrm{n} 0+1 / \mathrm{n} 1+1 / \mathrm{n} 2+1 / \mathrm{n} 3$

tss $=(y 1+y 2-y 3-y 0) / \operatorname{sqrt}(\operatorname{sum}(\mathrm{sy}) / \mathrm{dft}) / \operatorname{denf}$ 


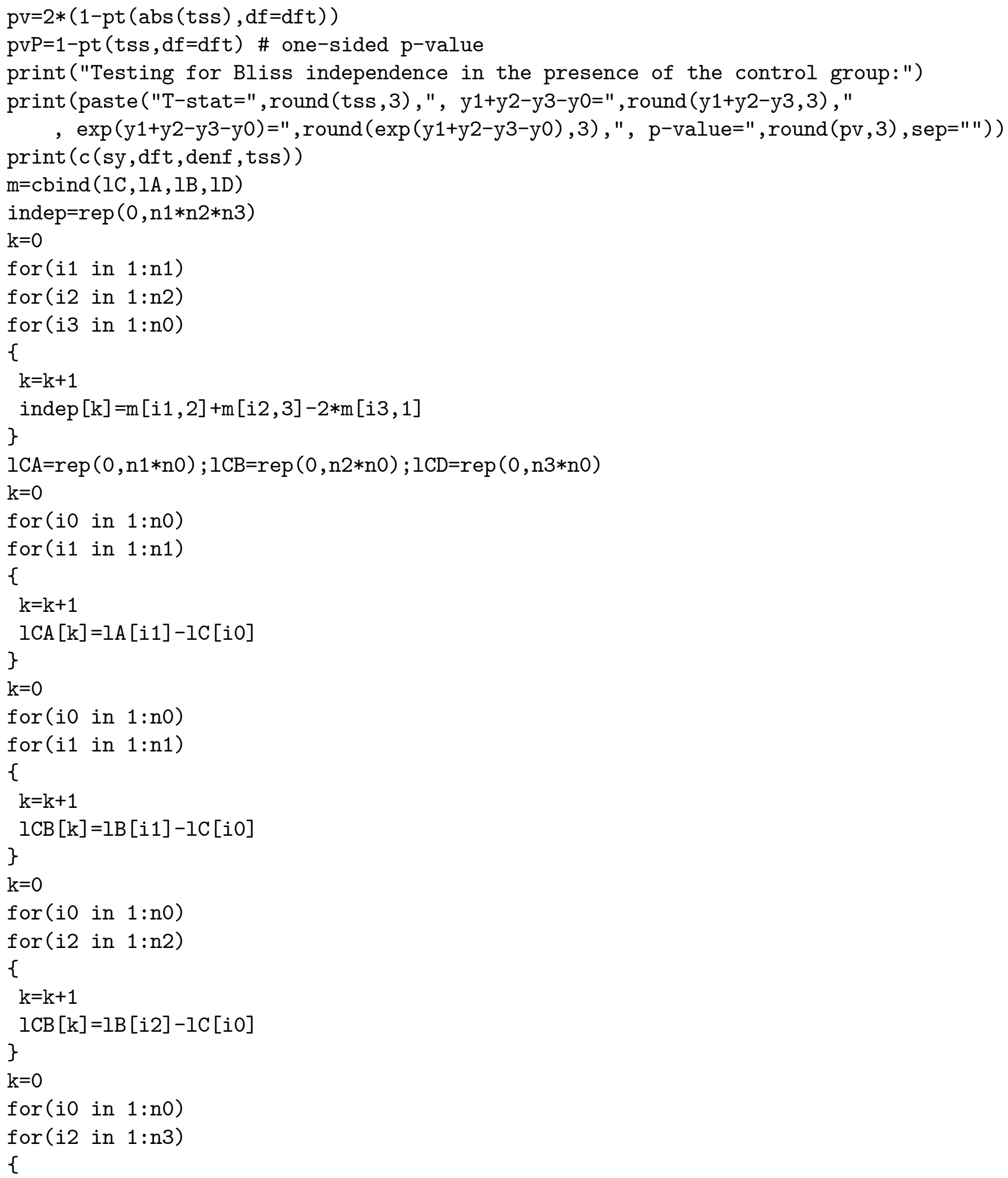




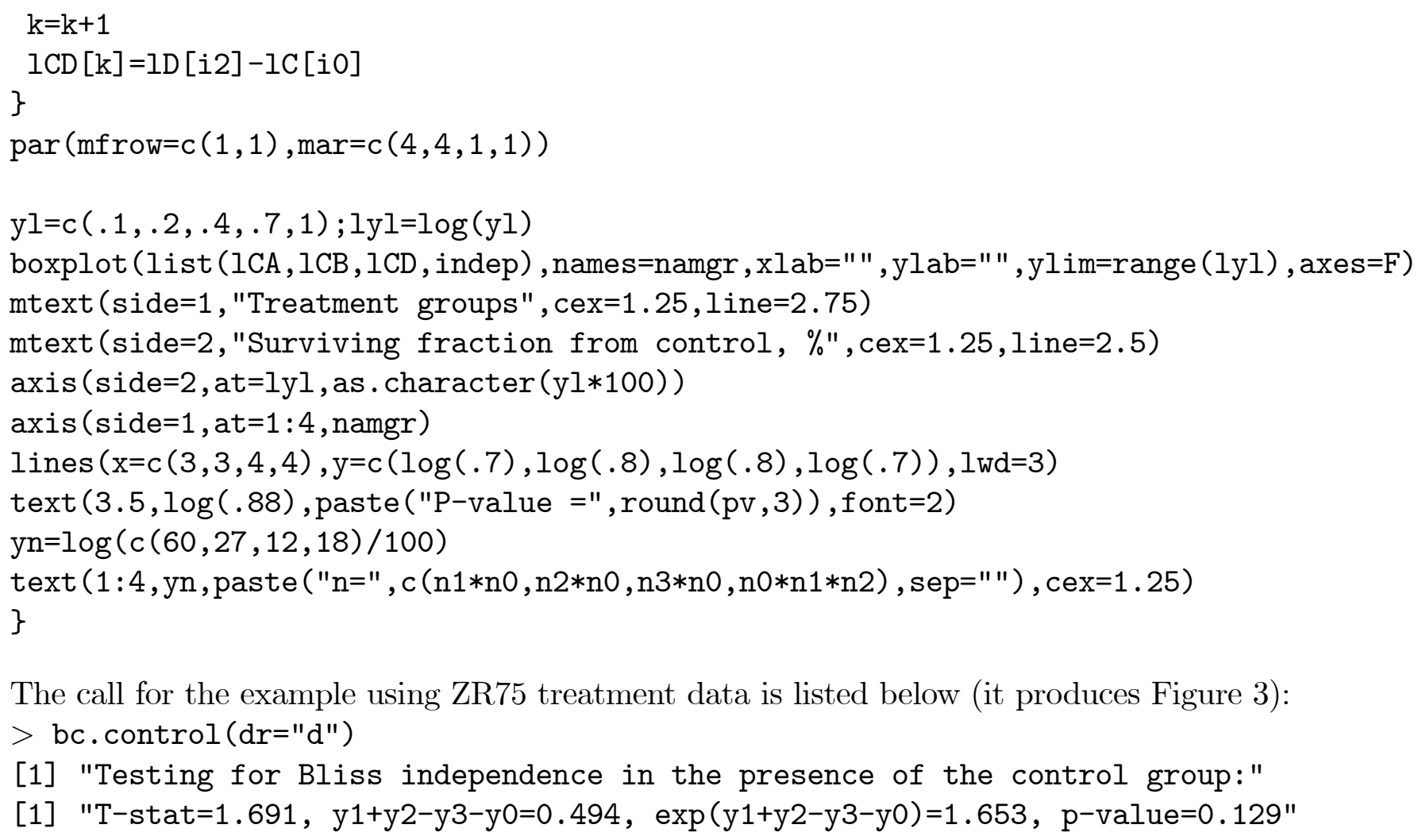

The call for the example using ZR75 treatment data is listed below (it produces Figure 3):

$>$ bc.control (dr="d")

[1] "Testing for Bliss independence in the presence of the control group:"

[1] "T-stat $=1.691, y 1+y 2-y 3-y 0=0.494, \exp (y 1+y 2-y 3-y 0)=1.653, p-v a l u e=0.129 "$

\section{Tumor growth experiments in vivo}

The tumor growth data in each group is estimated by linear mixed model with random intercept (mice-specific baseline tumor volume) using function lme from the library nlme, as discussed in detail in Demidenko (2013). The following R code plots Figure 6, estimates the rate of slope (beta coefficients) and the one-sided $p$-value for synergy. Tumor volume is plotted on the log scale with th etickmarks on the original $\mathrm{mm}^{3}$ scale. Before calling the tum.growth function the data file (provided) MCF-7_April_2016.csv must be saved in the folder dr, " : \Projects $\backslash \backslash$ ToddMiller $\backslash \backslash$ where $d r$ is the user defined hard drive (the default is $c$ ).

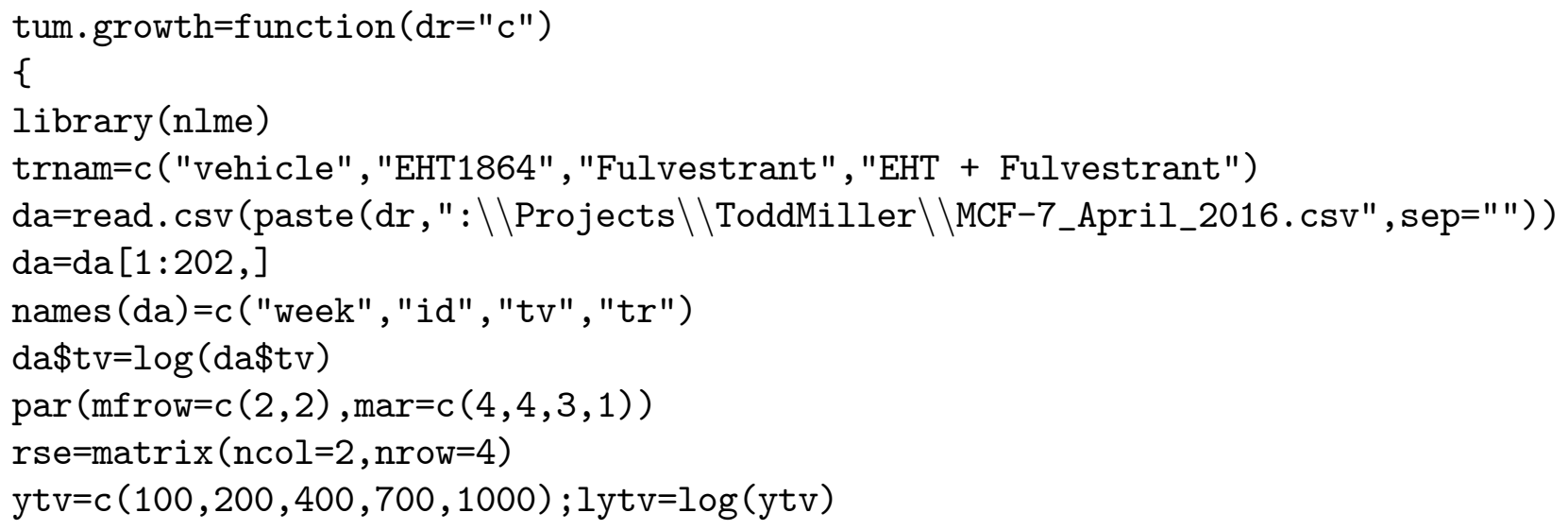




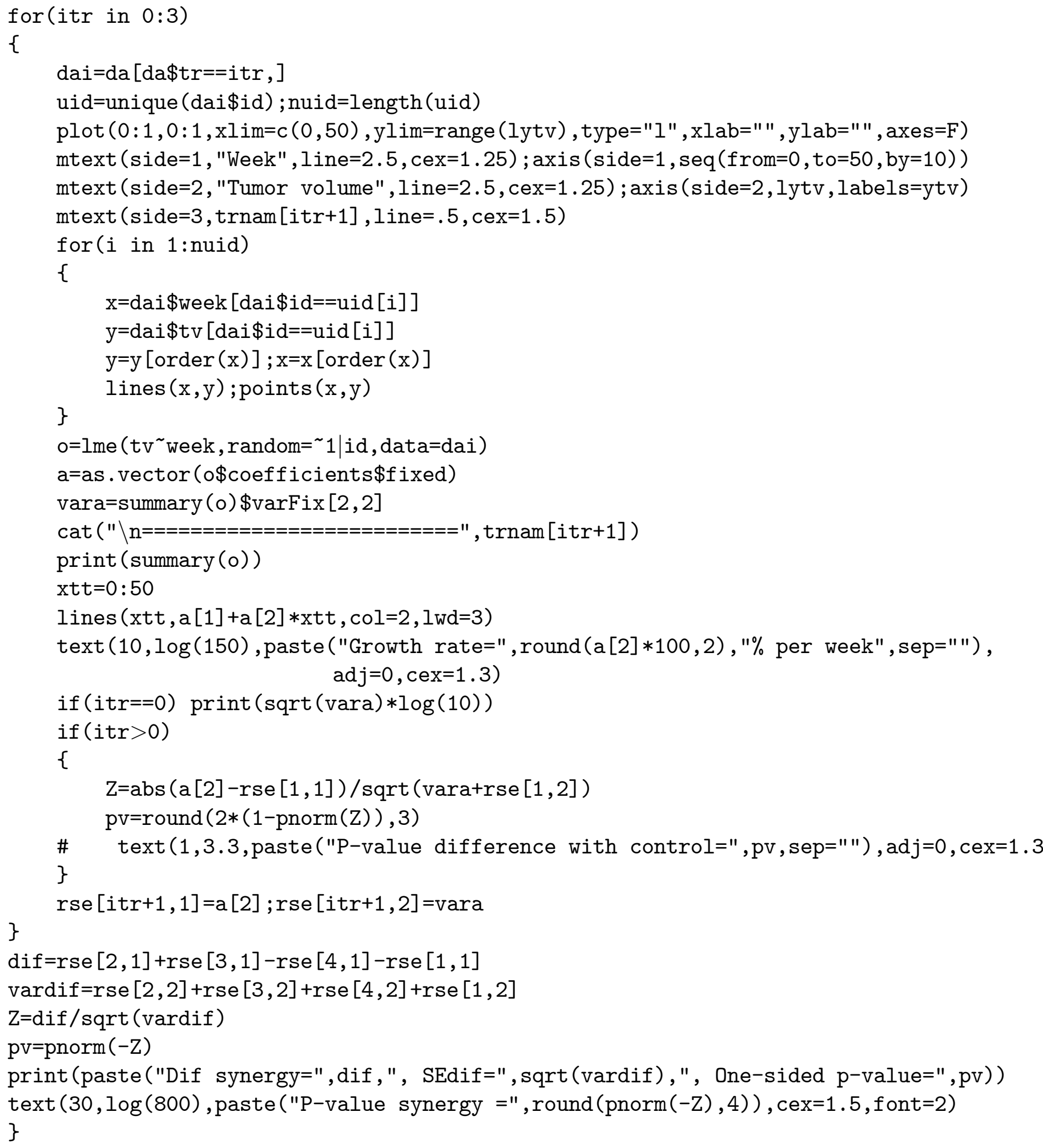

Below is the output of the call: 


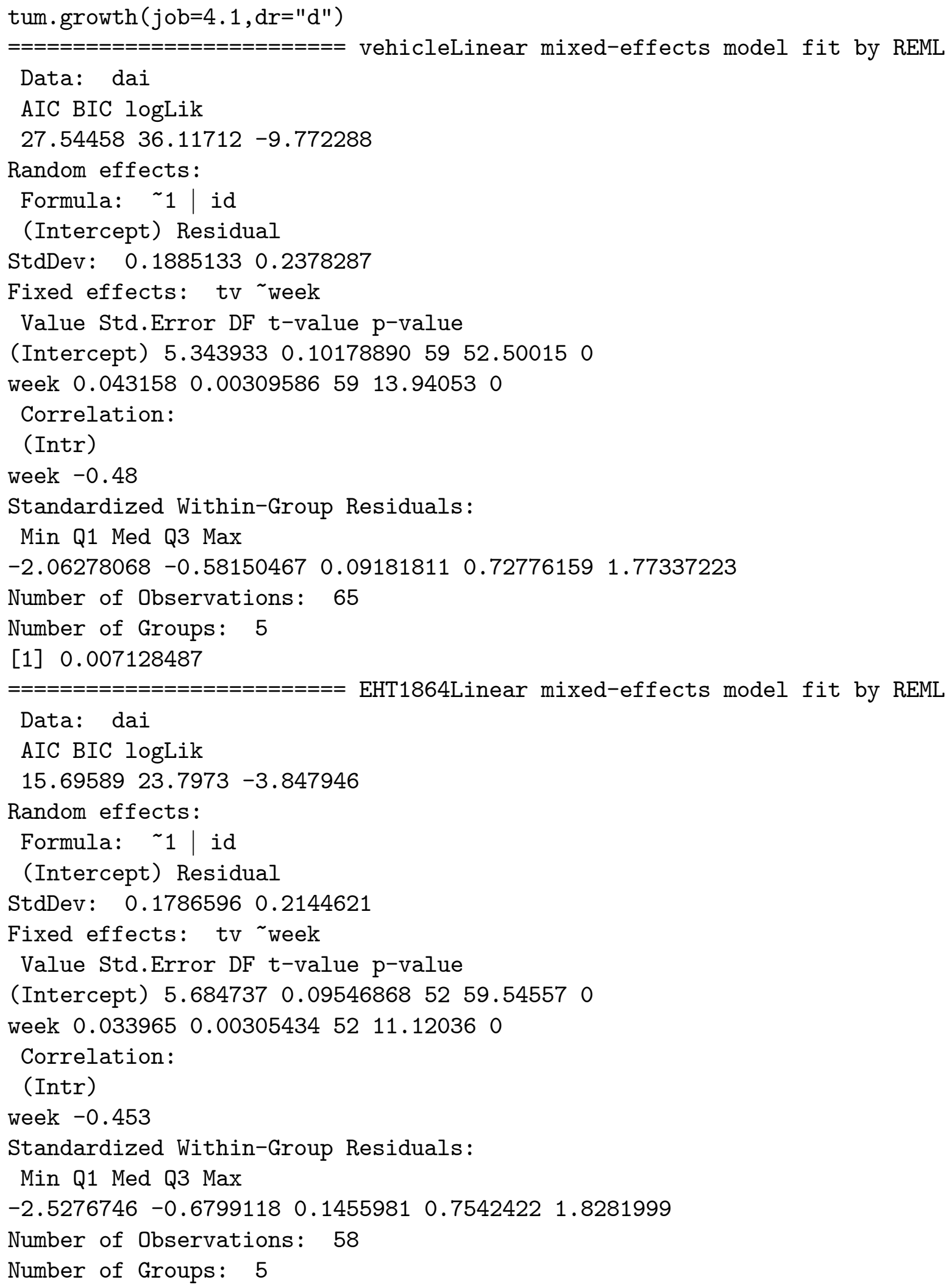




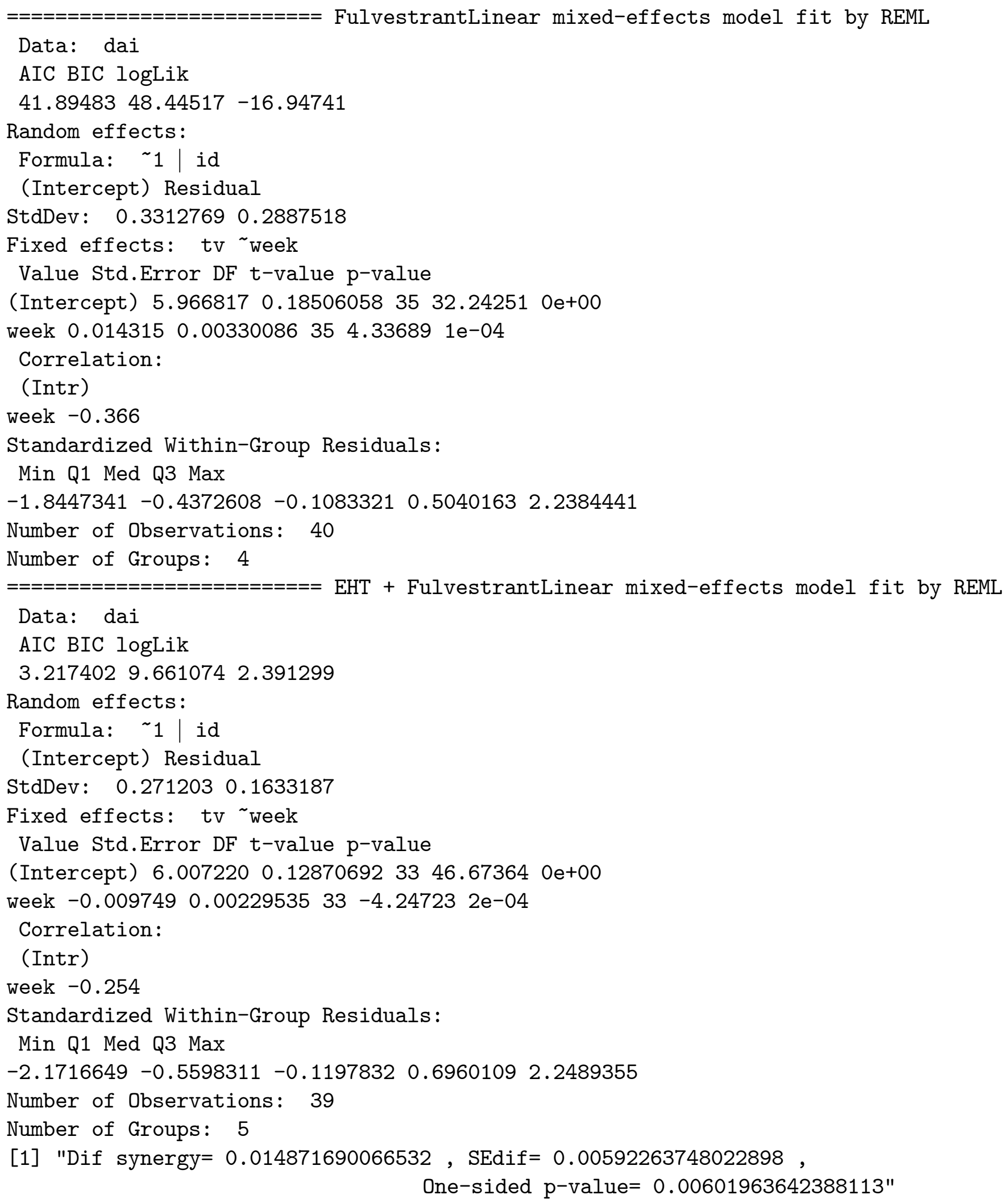




\section{Survival curves}

The following $\mathrm{R}$ function computes survival curve assuming Bliss independence and computes the $p$-value based on the logrank test using library survival (it must be installed before running this program). The call synergy.surv( $j \circ b=1)$ produces Figure 7 and the call synergy.surv( $j o b=3)$ produces Figure 8. The survival times ni, niip, and ip are taken from Larkin et al. paper (2015).

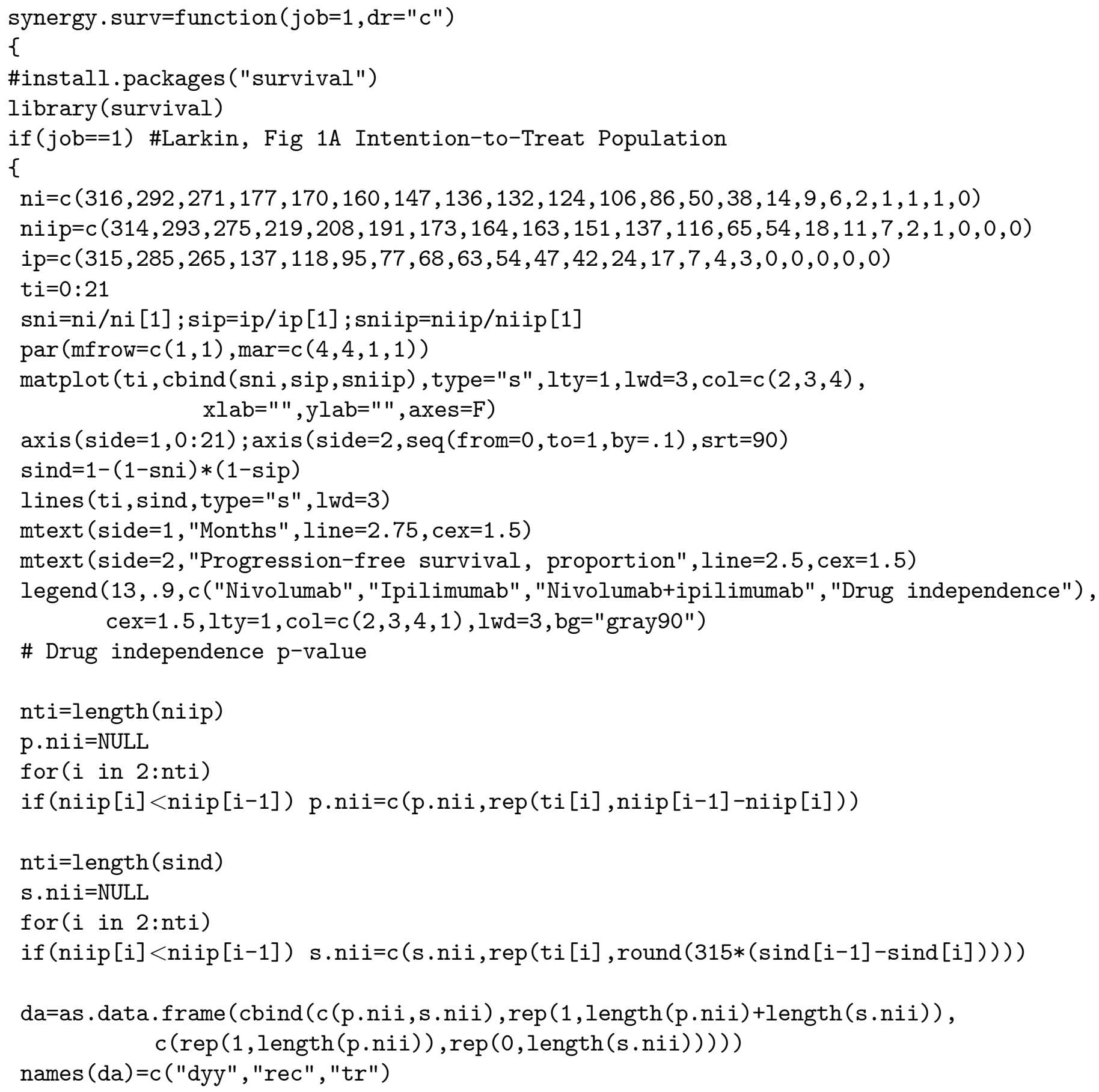




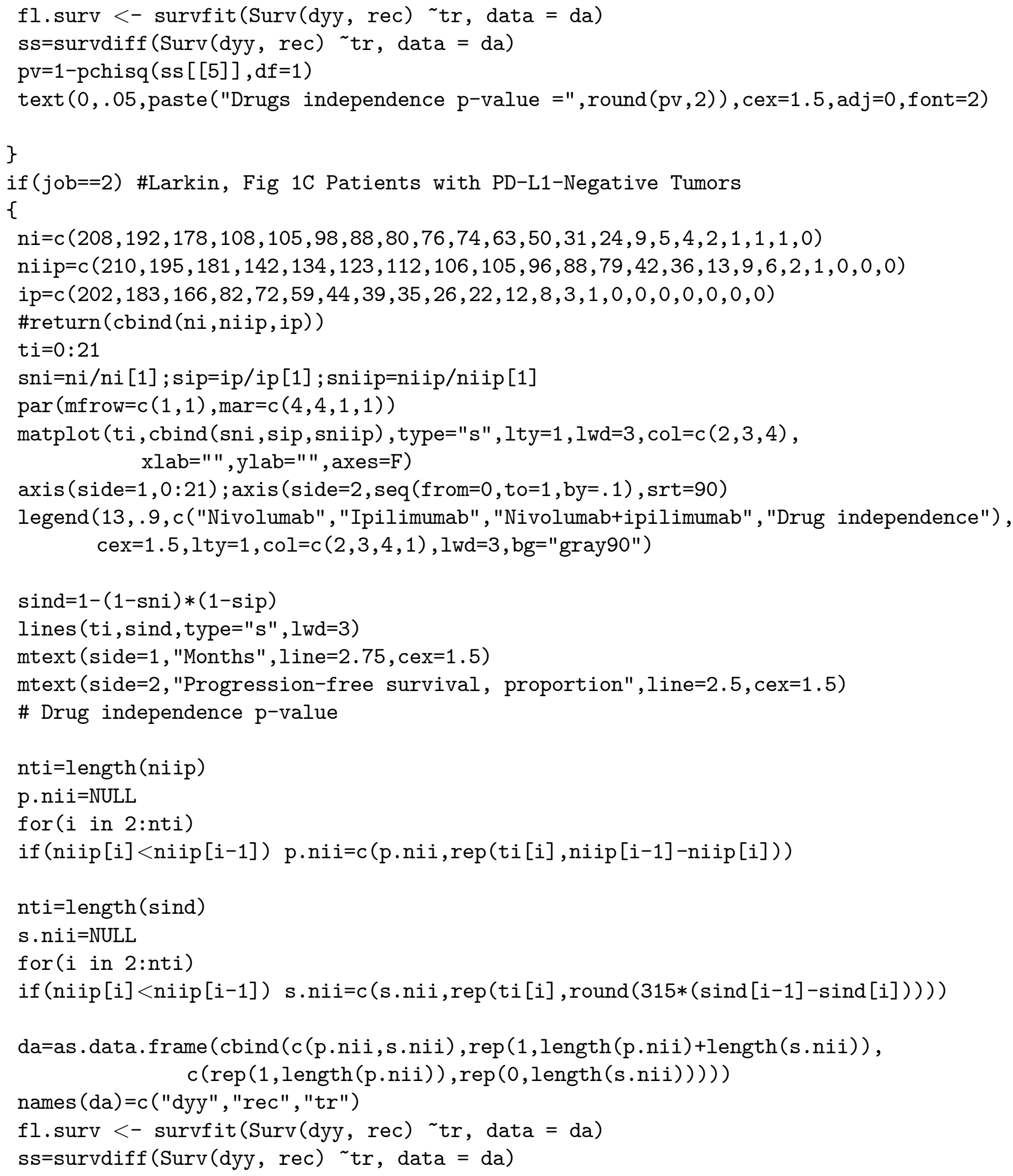


pv=1-pchisq (ss $[[5]], d f=1)$

text $(0, .05$, paste ("Drugs independence $p$-value $="$, round $(p v, 2)), \operatorname{cex}=1.5$, adj $=0$, font=2)

\}

\}

\section{Appendix. The two-drug copula and the $R$ code}

\subsection{Mathematical derivation and properties of the two-drug copula mortality function}

Let two drugs $A$ and $B$ have their own singe-agent mortality functions $M_{A}\left(\ln d_{A}\right)$ and $M_{B}\left(\ln d_{B}\right)$, where $d_{A}$ and $d_{B}$ individual doses. To simplify the notation denote $x=\ln d_{A}$ and $y=\ln d_{B}$. The mortality function $M(x)$, as any cdf, must (1) be an increasing function, (2) vanish when $x$ goes to $-\infty$, and (3) approach 1 when $x$ goes to $+\infty$. For example, the popular Hill mortality function $M(d)=\left(d / E C_{50}\right)^{m} /\left(1+\left(d / E C_{50}\right)^{m}\right)$ can be expressed on the log scale through the logit link, Demidenko (2017). The two-agent mortality function $M$ is built with the help of two probability concepts: the inverse normal cdf $\Phi^{-1}$ and the bivariate standard normal density with correlation coefficient $\rho$. The function $M$ which satisfies the properties formulated in the text, called the copula two-agent mortality function, is created as a double integral

$$
M(x, y ; \rho)=1-\int_{\Phi^{-1}\left(M_{A}(x)\right)}^{\infty} \int_{\Phi^{-1}\left(M_{B}(y)\right)}^{\infty} \phi(u, v ; \rho) d u d v
$$

where

$$
\phi(u, v ; \rho)=\frac{1}{2 \pi \sqrt{1-\rho^{2}}} e^{-\frac{1}{2\left(1-\rho^{2}\right)}\left(u^{2}-2 \rho u v+v^{2}\right)}
$$

is the bivariate standard normal density with the correlation coefficient $\rho$.

Property \#2 follows from the fact that if $d_{B} \rightarrow 0$ then $y \rightarrow-\infty$ and therefore $M_{B}(y) \rightarrow 0$ which implies $\Phi^{-1}\left(M_{B}(y)\right) \rightarrow-\infty$. Thus the lower and the upper limits of the inner integral are $-\infty$ and $\infty$ and integration turns the bivariate normal density into a marginal density,

$$
M(x,-\infty ; \rho)=1-\int_{\Phi^{-1}\left(M_{A}(x)\right)}^{\infty} \frac{1}{\sqrt{2 \pi}} e^{-\frac{1}{2} u^{2}} d u=1-\left(1-\Phi\left(\Phi^{-1}\left(M_{A}(x)\right)\right)\right)=M_{A}(x),
$$

because $\Phi(x)=\int_{-\infty}^{x}(2 \pi)^{-1 / 2} e^{-\frac{1}{2} u^{2}} d u$ and $\Phi\left(\Phi^{-1}\left(M_{A}(x)\right)\right)=M_{A}(x)$. The same proof works for drug $B$. This means that the copula two-agent mortality function turns into a single-agent mortality function in the absence of the other drug.

Property \#3 means that parameter $\rho$ controls the drug interaction. When $\rho=0$ two drugs act independently which means that function (1) turns into

$$
M(x, y ; 0)=1-\left(1-M_{A}(x)\right)\left(1-M_{B}(y)\right)
$$


the Bliss definition of independence. Indeed,

$$
\begin{aligned}
& M(x, y ; 0) \\
= & 1-\int_{\Phi^{-1}\left(M_{A}(x)\right)}^{\infty} \int_{\Phi^{-1}\left(M_{B}(y)\right)}^{\infty} \frac{1}{2 \pi} e^{-\frac{1}{2}\left(u^{2}+v^{2}\right)} d u d v \\
= & 1-\left(\int_{\Phi^{-1}\left(M_{A}(x)\right)}^{\infty} \frac{1}{\sqrt{2 \pi}} e^{-\frac{1}{2} u^{2}} d u\right)\left(\int_{\Phi^{-1}\left(M_{B}(y)\right)}^{\infty} \frac{1}{\sqrt{2 \pi}} e^{-\frac{1}{2} v^{2}} d v\right) \\
= & 1-\left(1-\int_{-\infty}^{\Phi^{-1}\left(M_{A}(x)\right)} \frac{1}{\sqrt{2 \pi}} e^{-\frac{1}{2} u^{2}} d u\right)\left(1-\int_{-\infty}^{\Phi^{-1}\left(M_{B}(y)\right)} \frac{1}{\sqrt{2 \pi}} e^{-\frac{1}{2} v^{2}} d v\right) \\
= & 1-\left(1-M_{A}(x)\right)\left(1-M_{B}(y)\right) .
\end{aligned}
$$

It is possible to prove that if $\rho>0$ we have synergy and if $\rho<0$ we have antagonism as stated in the property \#3.

Now we discuss numerical methods for computation our copula two-agent function. To facilitate computation (1) replace the lower finite limit of integration with the upper as follows:

$$
\begin{aligned}
& \int_{\Phi^{-1}\left(M_{A}(x)\right)}^{\infty} \int_{\Phi^{-1}\left(M_{B}(y)\right)}^{\infty} \phi(u, v ; \rho) d u d v=1-\int_{-\infty}^{\Phi^{-1}\left(M_{A}(x)\right)} \int_{-\infty}^{\Phi^{-1}\left(M_{B}(y)\right)} \phi(u, v ; \rho) d u \\
& -\int_{-\infty}^{\infty} \int_{-\infty}^{\Phi^{-1}\left(M_{B}(y)\right)} \phi(u, v ; \rho) d u+\int_{-\infty}^{\Phi^{-1}\left(M_{A}(x)\right)} \int_{-\infty}^{\Phi^{-1}\left(M_{B}(y)\right)} \phi(u, v ; \rho) d u d v
\end{aligned}
$$

and therefore (1) takes the form

$$
\begin{aligned}
& \int_{-\infty}^{\Phi^{-1}\left(M_{A}(x)\right)} d v \int_{-\infty}^{\infty} \phi(u, v ; \rho) d u+\int_{-\infty}^{\infty} d u \int_{-\infty}^{\Phi^{-1}\left(M_{B}(y)\right)} \phi(u, v ; \rho) d v \\
& -\int_{-\infty}^{\Phi^{-1}\left(M_{A}(x)\right)} \int_{-\infty}^{\Phi^{-1}\left(M_{B}(y)\right)} \phi(u, v ; \rho) d u d v .
\end{aligned}
$$

Finally, we obtain the following more convenient representation of (1):

$$
M(x, y ; \rho)=M_{A}(x)+M_{B}(y)-\int_{-\infty}^{\Phi^{-1}\left(M_{A}(x)\right)} \int_{-\infty}^{\Phi^{-1}\left(M_{B}(y)\right)} \phi(u, v ; \rho) d u d v .
$$

For example, the copula two-agent probit mortality function on the log scale takes the form

$$
M\left(d_{A}, d_{B}\right)=\Phi\left(\alpha_{A}+\beta_{A} \ln d_{A}\right)+\Phi\left(\alpha_{B}+\beta_{B} \ln d_{B}\right)-\int_{-\infty}^{\alpha_{A}+\beta_{A} \ln d_{A}} \int_{-\infty}^{\alpha_{B}+\beta_{B} \ln d_{B}} \phi(u, v ; \rho) d u d v
$$

where $\alpha_{A}, \beta_{A}, \alpha_{B}, \beta_{B}$, and $\rho$ are subject to estimation from the dose-response data. A technically similar to (2) but conceptually different probit-based two-drug dose-response model has been suggested earlier in Ashford and Sowden (1970) and Lesaffre and Molenberghs (1991); see also Fedorov 
and Leonov (2014). The technical difference is that our integration limit is lower and their integration limit is upper. The conceptual difference is that their model implies probabilistic independence when $\rho=0$, i.e. the two-drug function collapses to the product of single-drug functions while our model results in Bliss independence.

There are several ways to compute the double integral appeared in (2). First, one can use an $\mathrm{R}$ function pmvnorm from the package mvtnorm. Second, the double integral can be reduced to a one-dimensional integral using the formula

$$
\int_{-\infty}^{U} \int_{-\infty}^{V} \phi(u, v ; \rho)=\int_{-\infty}^{V} \Phi\left(\frac{U-\rho v}{\sqrt{1-\rho^{2}}}\right) \phi(v) d v
$$

where $\phi=\Phi^{\prime}$ is the density of the standard normal cdf. Using this representation we express (2) as

$$
M(x, y ; \rho)=M_{A}(x)+M_{B}(y)-\int_{-\infty}^{\Phi^{-1}\left(M_{B}(y)\right)} \Phi\left(\frac{\Phi^{-1}\left(M_{A}(x)\right)-\rho v}{\sqrt{1-\rho^{2}}}\right) \phi(v) d v .
$$

One-dimensional integration is a built-in function in many computer languages. For example, in $\mathrm{R}$ it is integrate, and therefore special packages such as mvtnorm are not needed if the above formula is used.

Proof of Theorem. The single integral representation formula (4) is used for the proof, where $x=\ln d_{A}$ and $y=\ln d_{B}$. (a) Without loss of generality we can assume that $x \geq y$ so that $M_{A}(x) \geq$ $M_{B}(y)$ because we assume that $M_{A}=M_{B}$. Then we need to prove that $M(x, y ; 1)=M_{A}(x)$. When $\rho \rightarrow 1$ the denominator of the argument of the $\Phi$ function in (4) goes to zero and the numerator converges to $\Phi^{-1}\left(M_{A}(x)\right)-v$. Therefore the argument of $\Phi$ converges to either $-\infty$ or $+\infty$ depending on the sign of $\Phi^{-1}\left(M_{A}(x)\right)-v$. Since $M_{B}(y) \leq M_{A}(x)$ we have $\Phi^{-1}\left(M_{B}(y)\right) \leq \Phi^{-1}\left(M_{A}(x)\right)$. Also we have $v \leq \Phi^{-1}\left(M_{B}(y)\right)$ since the right-hand side of this inequality is the upper limit of the integration. Combination of these two inequalities implies that the argument of function $\Phi$ converges to $+\infty$ and therefore the integral converges to $\int_{-\infty}^{\Phi^{-1}\left(M_{B}(y)\right)} \phi(v) d v=\Phi\left(\Phi^{-1}\left(M_{B}(y)\right)\right)=M_{B}(y)$. This means that in the limit $M(x, y ; 1)=M_{A}(x)+M_{B}(y)-M_{B}(y)=M_{A}(x)$. (b) When $\rho \rightarrow-1$ the argument of $\Phi$ converges to $-\infty$ or $+\infty$ depending on the sign of $\Phi^{-1}\left(M_{A}(x)\right)+v$ where $v \leq$ $\Phi^{-1}\left(M_{B}(y)\right)$, which implies that $\Phi^{-1}\left(M_{A}(x)\right)+v \leq \Phi^{-1}\left(M_{A}(x)\right)+\Phi^{-1}\left(M_{B}(y)\right)$. Consider the case when $M_{A}(x)+M_{B}(y) \leq 0.5$. Then both $\Phi^{-1}\left(M_{A}(x)\right)$ and $\Phi^{-1}\left(M_{B}(y)\right)$ are negative and therefore the argument of $\Phi$ in (4) converges to $-\infty$. This means that in the limit $M(x, y ;-1)=M_{A}(x)+M_{B}(y)$. The proof $M(x, y ;-1)=1$ when $M_{A}(x)+M_{B}(y)>1$ is omitted.

\section{Example: testing lethal effects of insecticides}

Below we list the $\mathrm{R}$ code for estimation of the bivariate copula dose-response model using nonlinear least squares (nls function). It requires library mvtnorm (install "mvtnorm" first). The single dose-response probit models are be estimated using glm . See more detail in [2].

\#install.packages ("mvtnorm")

library (mvtnorm) 


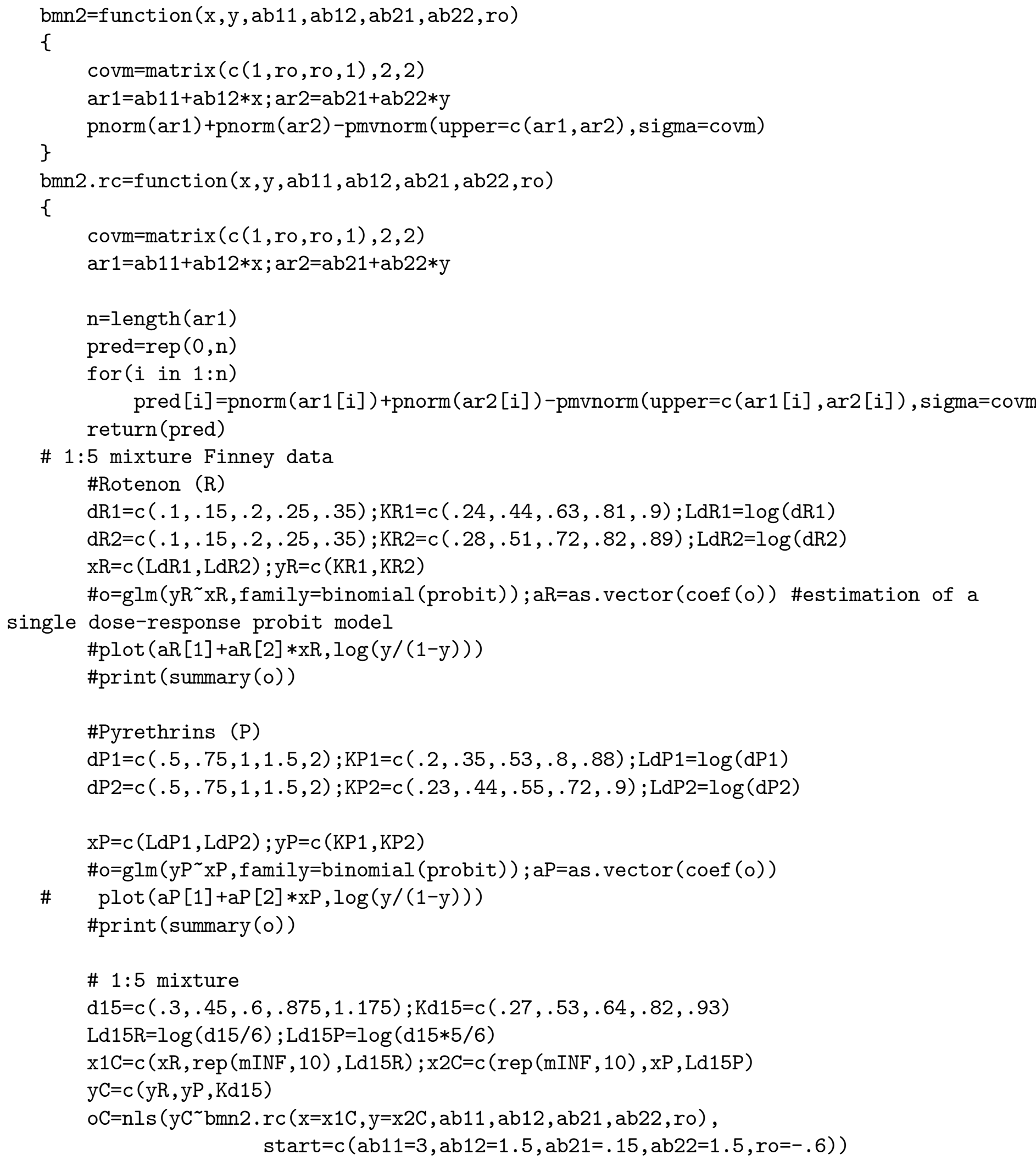

\# 1:5 mixture Finney data

\#Rotenon (R)

$\mathrm{dR} 1=\mathrm{c}(.1, .15, .2, .25, .35) ; \mathrm{KR} 1=\mathrm{c}(.24, .44, .63, .81, .9) ; \operatorname{LdR} 1=\log (\mathrm{dR} 1)$

$\mathrm{dR} 2=\mathrm{c}(.1, .15, .2, .25, .35) ; \mathrm{KR} 2=\mathrm{c}(.28, .51, .72, .82, .89) ; \mathrm{LdR} 2=\log (\mathrm{dR} 2)$

$\mathrm{xR}=\mathrm{c}(\mathrm{LdR} 1, \mathrm{LdR} 2) ; \mathrm{yR}=\mathrm{c}(\mathrm{KR} 1, \mathrm{KR} 2)$

$\# \mathrm{o}=\mathrm{glm}(\mathrm{yR} \sim \mathrm{xR}$, family=binomial (probit)) $\mathrm{aR}=\mathrm{as} \cdot \operatorname{vector}(\operatorname{coef}(\mathrm{o})$ ) \#estimation of a

single dose-response probit model

\#plot (aR [1] +aR [2]*xR, $\log (y /(1-y)))$

\#print (summary (o))

\#Pyrethrins (P)

$\mathrm{dP} 1=\mathrm{c}(.5, .75,1,1.5,2) ; \mathrm{KP} 1=\mathrm{c}(.2, .35, .53, .8, .88) ; \operatorname{LdP} 1=\log (\mathrm{dP} 1)$

$\mathrm{dP} 2=\mathrm{c}(.5, .75,1,1.5,2) ; \mathrm{KP} 2=\mathrm{c}(.23, .44, .55, .72, .9) ; \mathrm{LdP} 2=\log (\mathrm{dP} 2)$

$\mathrm{xP}=\mathrm{c}(\mathrm{LdP} 1, \mathrm{LdP} 2) ; \mathrm{yP}=\mathrm{c}(\mathrm{KP} 1, \mathrm{KP} 2)$

$\# \mathrm{o}=\mathrm{glm}(\mathrm{yP} \sim \mathrm{xP}$, family=binomial (probit) ); $\mathrm{aP}=\mathrm{as} \cdot \operatorname{vector}(\operatorname{coef}(\mathrm{o}))$

\# $\quad \operatorname{plot}(\mathrm{aP}[1]+\mathrm{aP}[2] * \mathrm{xP}, \log (\mathrm{y} /(1-\mathrm{y})))$

\#print (summary (o))

\# 1:5 mixture

$\mathrm{d} 15=\mathrm{c}(.3, .45, .6, .875,1.175) ; \mathrm{Kd} 15=\mathrm{c}(.27, .53, .64, .82, .93)$

$\mathrm{Ld15R}=\log (\mathrm{d} 15 / 6) ; \mathrm{Ld} 15 \mathrm{P}=\log (\mathrm{d} 15 * 5 / 6)$

$\mathrm{x} 1 \mathrm{C}=\mathrm{c}(\mathrm{xR}, \operatorname{rep}(\mathrm{mINF}, 10), \operatorname{Ld} 15 \mathrm{R}) ; \mathrm{x} 2 \mathrm{C}=\mathrm{c}(\mathrm{rep}(\mathrm{mINF}, 10), \mathrm{xP}, \mathrm{Ld} 15 \mathrm{P})$

$\mathrm{yC}=\mathrm{c}(\mathrm{yR}, \mathrm{yP}, \mathrm{Kd} 15)$

$\mathrm{oC}=\mathrm{n} 1 \mathrm{~s}(\mathrm{yC} \sim \mathrm{bmn} 2 . \mathrm{rc}(\mathrm{x}=\mathrm{x} 1 \mathrm{C}, \mathrm{y}=\mathrm{x} 2 \mathrm{C}, \mathrm{ab} 11, \mathrm{ab} 12, \mathrm{ab} 21, \mathrm{ab} 22, \mathrm{ro})$, start $=c(a b 11=3, a b 12=1.5, a b 21=.15, a b 22=1.5, r o=-.6))$ 


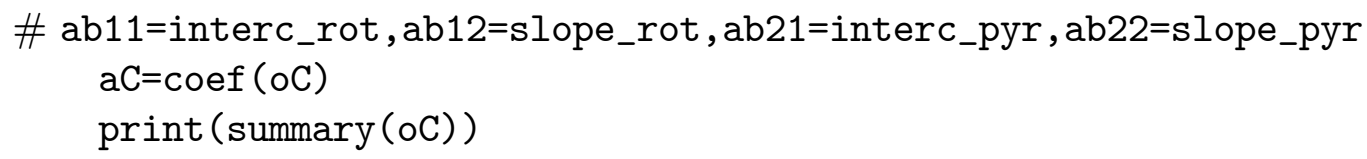

\section{References}

Demidenko, E. (2103). Mixed Models: Theory and Applications in R. Hoboken: Wiley.

Demidenko, E., Glaholt, S.P., Kyker-Snowman, E., Shaw, J.R., Chen, C.Y. (2017). Single toxin dose-response models revisited. Toxicology and Applied Pharmacology 314, 12-23.

Larkin, J., Chiarion-Sileni, V., Gonzalez, R.,et al. (2015). Combined nivolumab and ipilimumab or monotherapy in untreated melanoma. New England Journal of Medicine 373, 23-34.

R Core Team (2017). R: A language and environment for statistical computing. R Foundation for Statistical Computing, Vienna, Austria. URL https://www.R-project.org/. 